probably not linked to a genetic susceptibility to epilepsy. (Verrotti A, Trotta D, Salladini C et al. Photosensitivity and epilepsy: a follow-up study. Dev Med Child Neurol 2004;46:347351). (Respond: Alberto Verrotti MD, PhD, Department of Medicine, Section of Pediatrics, University of Chieti-Ospedale Policlinico, Via dei Vestini 5, 66100 Chieti, Italy).

COMMENT. Photosensitive epilepsy is defined as recurrent seizures in photosensitive patients, with EEG-PPRs in response to intermittent photic stimulation. According to several references cited by the authors, photosensitivity (PS) occurs in 2 to $20 \%$ of patients with epilepsy, and may also be present in non-epileptic individuals. PS can occur in any type of epileptic disorder and is not a specific epileptic syndrome. Pure PS epilepsy is characterized by generalized paroxysmal epileptiform discharges exclusively provoked by flicker, usually in adolescence, predominantly females. PS epilepsy has a good prognosis, independent of disappearance or persistence of PS

Short-term outcome of Pokemon phenomenon and TV-induced seizures was determined at Nagoya University, Japan (Ishiguro Y, Takada H, Watanabe K et al. A follow-up survey on seizures induced by animated cartoon TV program "Pocket Monster." Epilepsia April 2004;45:377-383). Of 103 patients with epileptic seizures during the TV program, 25 (24\%) had unprovoked seizures before the incident (Epilepsy group), and 78 (76\%) did not (Nonepilepsy group). Twenty-three (22\%) had seizures after the incident, 15 visually induced. Patients in the Epilepsy group had more seizure recurrences (both unprovoked or visually induced) than the Non-epilepsy group ( $56 \%$ vs $9 \%$; p $<0.0001)$. PPRs on EEG were present in $45(46 \%)$ patients, and spontaneous epileptiform discharges in $49(50 \%)$. PPRs were not correlated with recurrence of seizures, whereas spontaneous epileptiform discharges were significantly correlated $(34 \%$ vs $8 \% ; \mathrm{p}<0.01)$. Of patients with seizures during the TV incident, $70(68 \%)$ had no seizures before or during the 3 year follow-up. Color changes, specifically red, rather than luminance was found to be the critical seizure-inducing factor in the TV scene, prompting color restrictions in TV cartoon programs in Japan.

For reports and commentaries on visually-induced seizures, including video game seizures and pattern sensitive seizures, see Progress in Pediatric Neurology III, PNB Publishers, 1997;64-67.

\title{
SLEEP DISORDERS
}

\section{DIAGNOSIS AND MANAGEMENT OF CHILDHOOD NARCOLEPSY}

The clinical and polysomnographic presentation of 12 published cases of narcolepsy with onset from early infancy to 8 years is reviewed at Victoria University, Melbourne, and Flinders Medical Centre, Adelaide, Australia. Diagnosis was made at ages 2 years to 12 years. Clinical presentations included excessive daytime sleepiness (EDS) in all cases, cataplexy in 6, behavioral problems and/or inattention in 6, hypnogogic hallucinations in 3 , and sleep paralysis in 1. Alternate diagnoses considered in 5 patients were epilepsy (3), comorbid ADHD and ODD (2), developmental delay (2), obstructive sleep apnea (1), and Munchausen syndrome by proxy (1). Sleepiness in narcolepsy persists despite adequate nocturnal sleep and it occurs in inappropriate environments, while eating, watching TV, playing, and even walking. Persistence of daytime napping after 4 years of age may be an 
early indicator. EDS is mistaken for a 'problem behavior' on entry to school, when napping is not permitted. Narcolepsy presents in response to school based concerns about EDS and inattentiveness in $78 \%$ of childhood cases over 5 years (Guilleminault, 1998). Hypersomnolence may also present as hyperactivity and irritability (Wise, 1998). Like EDS, cataplexy is rarely classic in presentation. Abnormal clumsy behavior, falls or bumps, dropping and breaking of objects, 'drop attacks' occur, but rarely in association with laughter or excitement. In one report of 77 childhood cases, cataplexy developed on average within 1 year after EDS symptoms, and the time lag is considerably shorter than in adults. The reporting of sleep paralysis and hallucinations is largely dependent on the age of the child. Estimates suggest that in children with narcolepsy at a mean age of 9 years, $97 \%$ have experienced EDS, $80 \%$ cataplexy, $39 \%$ hallucinations and $29 \%$ sleep paralysis, $13 \%$ having the full tetrad of symptoms (Challamel, 1994), a finding similar to that in adults. Differential diagnoses include: vaso-vagal or cardiogenic syncope, nocturnal insomnia with obstructive sleep apnea, Pickwickian syndrome, Chiari type 1, hypothalamic dysfunction or tumor, Prader-Willi syndrome, disordered circadian rhythm, atonic seizures, Coffin-Lowry syndrome, pontine tumor, and Niemann Pick disease (diagnosed in 12 of 97 cases of narcolepsy (Challamel, 1994). Genetic markers HLA-DR2 and DQB1 have been associated with narcolepsy but genetic penetrance is low. Recent work has pointed to a degeneration of the neuropeptide orexin in the hypothalamus as the cause. A definitive diagnosis in adults is made with attacks of EDS, cataplexy, and/or sleep onset REM (SOREM) periods measured using polysomnography and the Multiple Sleep Latency Test (MSLT). A mean sleep latency of $<5$ minutes and/or 2 or more sleep onset REM periods is diagnostic. The MSLT is unreliable in children below 8 years, although polysomnography may help in exclusion of nocturnal sleep disorders. In treatment, stimulants are only partially effective, tricyclics (clomipramine) have been advocated for cataplexy, and a daytime nap schedule is effective in preschoolers. Psychosocial issues are common including emotional lability, aggressive behaviors, social isolation, bullying, academic underachievement and depression. In a cohort of 40 children aged 7 and older, $90 \%$ described a sense of shame and $20 \%$ had reactive depression (Guilleminault, 1998). School transition is a critical period of adjustment, requiring psychosocial intervention strategies. (Hood BM, Harbord MG. Narcolepsy: diagnosis and management in early childhood. J Pediatr Neurol 2004;2(2):65-71). (Respond: Dr Bernadette Hood, Department of Psychology, Victoria University, PO Box 14428 MCMC 8001, Melbourne, Australia).

COMMENT. Narcolepsy in young children is usually atypical and frequently misdiagnosed. Diagnosis is based primarily on clinical symptoms, and polysomnography is usually inconclusive. Medications are often less effective than in adults, and psychosocial issues on entering school need wider recognition and attention.

\section{INFECTIOUS DISEASES}

\section{CHANGING CLINICAL FEATURES OF SUBACUTE SCLEROSING PANENCEPHALITIS (SSPE)}

The early clinical features, age at onset, and relation to immunization of 32 patients (24 males, 8 females) with SSPE are reported from Grant Medical College, Mumbia, India. 